\title{
Art and Exoticism. An anthropology of the yearning for authenticity de Paul VAN DER GRIJP
}

\section{Raymond MAYER}

\section{OpenEdition}

\section{Journals}

Édition électronique

URL : http://journals.openedition.org/jso/5981

DOI : 10.4000/jso.5981

ISSN : $1760-7256$

Éditeur

Société des océanistes

\section{Édition imprimée}

Date de publication : 15 décembre 2009

Pagination : 339-340

ISBN : 978-2-85430-026-0

ISSN : 0300-953x

\section{Référence électronique}

Raymond MAYER, "Art and Exoticism. An anthropology of the yearning for authenticity de Paul VAN DER GRIJP », Journal de la Société des Océanistes [En ligne], 129 | juillet-décembre 2009, mis en ligne le 15 décembre 2009, consulté le 21 septembre 2020. URL : http://journals.openedition.org/jso/5981 ; DOI : https://doi.org/10.4000/jso.5981 
Van Der GriJp Paul, 2009. Art and Exoticism. An anthropology of the yearning for authenticity, Berlin, Lit Verlag, 358 p., bibliogr., index.

Le nouvel ouvrage de Paul Van der Grijp, professeur d'anthropologie dont l'université Lumière-Lyon 2 s'est attaché les services, est une excellente plongée dans un «penchant culturel» qui semble aller de soi et que nous hésitons à remettre en cause : notre appétence pour l'exotique et notre souci pour de l'authentique. Il complète l'approche des arts déjà développée dans un livre antérieur, écrit dans la perspective des collectionneurs, Passion and Profit: Towards an Anthropology of Collecting (2006). Entre « l'anthropologie des collecteurs» et "l'anthropologie de la recherche d'authenticité », on devine que la seconde prolonge, en l'affinant, la quête de l'objet d'art et des désirs avoués ou inavoués qu'elle suscite et comble tout à la fois.

Question distance, nous sommes servis, puisque l'angle d'attaque est d'une certaine manière océaniste, l'ouvrage s'ouvrant et s'achevant sur les appréciations portées par Victor Segalen sur son expérience polynésienne. Au début de son livre, Paul Van der Grijp tient les appréciations du médecin de marine arrivé en 1903 à Tahiti, pour des réflexions premières, de type anthropologique, non ethnocentriques, sur la question de l'altérité culturelle. Même s'il ne critique jamais son insertion dans le système colonial français, Victor Segalen lui apparaît comme une sorte de premier anthropologue qui ne mystifie ni n'idéalise les cultures qu'il rencontre avec empathie. Quelques extraits de sa biographie serviront donc de moteur au dessein exploratoire de l'ouvrage. Paul Van der Grijp a même repris à son avantage un projet explicite, mais sans cesse différé, de Victor Segalen, qui était de publier un ouvrage sur l'exotisme.

Celui-ci parut finalement à titre posthume - assez tardivement puisqu'il fallut attendre l'année 1978, mais avec un certain succès puisqu'il fut réédité dès 1986, sous le titre d'origine Essai sur l'exotisme. Le projet de Van der Grijp est quelque peu différent : il fait de Segalen une sorte de ludion de son livre, le consacrant guide imaginaire de son exploration conceptuelle pour parcourir le vaste espace des amateurs du « regard éloigné » et des collectionneurs de tous horizons. Ce faisant, son livre - que l'on n'ose qualifier de « catalogue » car il ne comporte malheureusement aucune illustration, à l'exception de la page de couverture qui nous présente en noir et blanc un masque facial anonyme photographié par l'auteur nous entraîne dans une revue complète d'un siècle de peinture occidentale et d'expériences artistiques des antipodes passées au prisme des influences exotiques et des apports extérieurs. Mais ce parcours est luimême un prétexte pour explorer le concept d'exotisme et rationaliser celui d'authenticité. Pour parvenir à ses fins, l'auteur pratique le travelling oblique, s'arrêtant à plusieurs gares et ancrant dans plusieurs ports.

Le livre s'ouvre ainsi sur le mouillage du navire de Victor Segalen à Java (p. 7) - sans rappeler expressément la date de cette escale de retour : est-ce pour installer l'auteur des Immémoriaux dans une sorte de pérennité paradigmatique ? - et il s'achève, pour définir l'exotisme, sur un dépassement de la conscience d'altérité telle qu'imputée au même auteur (p. 318). Entre ces deux repères, Paul Van der Grijp procède à des rappels périodiques de son auteur de référence, à la fois pour baliser le terrain conceptuel et pour débusquer l'exotisme sur le marché de l'art qu'il répertorie au fur et à mesure qu'il nous le fait découvrir au loin et redécouvrir à domicile.

Les six premiers chapitres, dont le chapitre introductif, sont alloués à une analyse conceptuelle dans laquelle le concept d'exotisme est successivement rapproché, sans s'y dissoudre, des concepts de différence culturelle (chap. 1), de globalisation (chap. 2), d'antiquité, de paradis et de rapport à la nature (chap. 3), de civilisation et d'esthétique (chap. 4), de collection et de distance (chap. 5), et enfin d'érotisme (chap. 6). Les arguments théoriques sont entrelacés d'observations concrètes et d'anecdotes vivantes. On appréciera ainsi aux pages 65-69 le taux des trocs pratiqués, sur leurs terrains respectifs, par Malinowski et Firth pour l'acquisition d'objets « exotiques » contre des objets manufacturés (hameçons, pipes et couteaux). Ou encore l'érotisme induit par les cartes postales exotiques d'époque (pp. 81-87).

Par allusions furtives, nous retrouvons ensuite Victor Segalen déambulant à travers une histoire de l'art comme témoin théorique de l'évolution des conceptions artistiques qui se bâtissent à coups d'inspirations exotiques. Comme Moussorgski dans sa visite des « Tableaux d'une exposition », Paul Van der Grijp sait reconstituer (chap. 7 à 11) l'atmosphère des mouvements artistiques européens qui se sont succédé à partir de l'orientalisme d'Eugène Delacroix jusqu'aux peintures en extérieur des impressionnistes et aux ateliers abondant en arts nègres des fauves, cubistes, surréalistes et dadaïstes ultérieurs. Chaque halte de l'auteur devant les œuvres d'un Millet, Courbet, Pissarro ou Gauguin (chap. 8) est l'occasion de mesurer ce que ces « inventions » artistiques doivent à l'inspiration de modèles « exotiques ». La biographie de Victor Segalen reprend à l'année 1903 (p. 131), au moment de son débarquement aux Marquises où Gauguin vient de décéder, et à la période où il acquiert sept peintures de cet artiste qui n'est pas encore considéré comme un maître, aux enchères improvisées de Papeete. Le propos de Paul de Van der Grijp est alors d'insérer l'histoire de Segalen dans l'histoire d'un Gauguin à l'exotisme non encore reconnu.

La visite $\mathrm{du} \mathrm{xx}^{\mathrm{e}}$ siècle de la peinture occidentale s'arrête un bon moment devant Picasso (chap. 9) et devant le cours des écoles formelles de l'époque (chap. 10). L'étude est érudite et fouillée, se faisant plaisir, entre autres, à nous conter la genèse au jour le jour des Demoiselles d'Avignon. Mais Van der Grijp y ajoute de son pinceau d'ethnographe, en nous conviant, pour clore cette revue européenne, à trois études de cas d'artistes contemporains du sud de la France (chap. 11) qui apportent au débat leur propre conception de l'exotisme.

À partir de là, le livre opère une véritable bifurcation dans l'analyse, en croisant les regards sur des terrains non occidentaux, et en nous amenant à juger de l'exo- 
tisme en sens alterné. Un balayage dialectique est effectué sur les influences réciproques entre l'Orient et l'Occident au Japon, en Chine et en particulier à Taïwan (chap. 11). Dans un genre où il excelle, l'auteur convoque ensuite d'autres études de cas, principalement empruntées aux terrains tongiens (chap. 12), fidjiens et néo-zélandais (chap. 13), où il s'est rendu sur les marchés locaux. Des artisans communs ou des artistes émergents font part de leurs critères d'authenticité et d'inspiration artistique. Pour connaître les canons qu'ils appliquent à leurs productions artistiques ainsi que leurs sources d'inspiration, Paul Van der Grijp prend langue à Tonga avec Lopati, Ofa, Tevita, Feleti, Kaufili et Tomasi (pp. 208-212). Nous apprenons ainsi que leur première préoccupation est l'accès à un marché approprié pour commercialiser leurs œuvres. Leur définition de «l'authenticité 》 des œuvres est référée sans ambiguïté aux traditions séculaires : «faire du tongien », « faire du fidjien »; mais la pratique de l'innovation n'est pas interdite pour autant. "Je veux que chaque pièce soit différente, déclare Lopati ; je n'aime pas me répéter !» (p. 252). Ce qui en dit long sur la capacité à innover dans des productions censées s'inspirer de modèles traditionnels. Le livre examine le cas particulier des matières premières (ivoire, matières osseuses, corail noir) qui font intervenir l'obligation de respecter les conventions internationales concernant les espèces protégées (chap. 14 et 15). Celles-ci n'empêchent ni les amateurs de satisfaire leur goût pour l'exotisme, ni les artistes de continuer à faire face à la demande.

Victor Segalen réapparaît en conclusion (p. 318), au moment à l'auteur pose le bilan de son investigation antipodique, au terme du parcours qui nous aura amenés du bateau de Polynésie jusqu'aux galeries parisiennes, tout en nous ayant introduit aux ateliers outremer de différents continents et sous-continents, de l'Asie à l'Océanie notamment. La définition multicritère initiale de l'exotisme selon Victor Segalen (p. 9) qui a servi d'amorce à cette exploration tous azimuts, est alors passée au crible des exemples et des contreexemples qui ont nourri l'argumentaire du livre. Paul Van der Grijp est en mesure de proposer à son tour six critères définissant l'authenticité (pp. 315-316), reconnaissant que le critère de la «qualité artistique » est l'un des plus difficiles à valider. Quoi qu'il en soit, il refuse plusieurs réductions qui lui semblent attachées au concept d'exotisme de la version Victor Segalen. La seule reconnaissance de la distance culturelle et de l'incompréhension culturelle, telles que stipulées par l'auteur de référence, ne lui suffit pas. Car il lui manquerait la contrepartie identitaire qui mérite au minimum une réciprocité de perspectives, celle qui est justement fournie par une approche de type ethnographique. De même, la réduction au «primitivisme » le priverait de sa dimension inconsciente, qui nécessite sans aucun doute le recours à une approche de type psychanalytique. Enfin le rapprochement avec le concept d'érotisme lui fait dire qu'il faudrait inclure une approche de genre dans la conception de l'exotisme. Et de conclure par l'obligation d'approfondir l'examen des motivations sous-jacentes au concept. Manière de dire que, malgré la profusion des exemples déjà mis en scène dans l'ouvrage, la porte est ouverte pour d'autres investigations conceptuelles aussi circonstanciées.

Il est évidemment dommage que l'ouvrage traite de son sujet « en aveugle », puisqu'aucune image ne vient corroborer le discours roboratif de l'auteur. C'est probablement dire sa volonté de se situer à un niveau plutôt théorique et d'être plus proche d'un essai philosophique que de celui d'un critique d'art. Mais si les productions de l'histoire de l'art occidental qui jalonnent sa démonstration nous sont connues, celles des « jeunes écoles » artistiques du Pacifique, de Taiwan, de Fidji, de Tonga ou de Nouvelle-Zélande ne nous le sont pas, et il est donc difficilement concevable que nous analysions des formes, des emprunts et des pratiques éloignées, en quelque sorte par procuration. Sur ce point, il eût été préférable de travailler sur pièce et de montrer toutes les productions artistiques utiles à la démonstration. Les études de cas auraient donc gagné à être illustrées, au sens que l'impression « esthétique » produite par une œuvre est médiatisée par sa mise en présence effective, ne serait-ce que par un dispositif iconographique minimal. Cette réserve faite, ce livre est suffisamment riche en documentation écrite et en discussion théorique pour susciter un intérêt qui ne s'arrête pas à sa seule lecture.

Raymond MAYER, Université Omar Bongo de Libreville et Université de Lyon 2

Bowden Ross, 2006. Creative Spirits. Bark Painting in the Washkuk Hills of North New Guinea, Melbourne, Oceanic Art Pty Ltd, x-204 p., tableaux, bibliogr., index, carte, 137 photographies noir et blanc et couleur.

MÉlandri Magali et Maxime Rovere (s. d.), 2008. Rouge kwoma. Peintures mythiques de NouvelleGuinée, Paris, Réunion des musées nationauxMusée du quai Branly, 96 p., bibliogr., carte, 52 illustrations en couleur.

Les Kwoma sont des habitants des collines, comme ils se nomment eux-mêmes, vivant sur la rive gauche du Sépik, à environ $200 \mathrm{~km}$ à vol d'oiseau de l'embouchure de ce fleuve, non loin d'Ambunti, où un poste de patrouille australien fut établi dès 1924. Leurs collines, les Washkuk Hills, dominent de 250 mètres tout au plus le cours du fleuve, qui n'est alors qu'à 45 mètres au-dessus du niveau de la mer. Moins d'un millier en 1936 selon le dénombrement de Whiting (1941: 5), entre 1700 et 2000 au début des années 1970 (Kaufmann, 1979 : 311 ; Bowden, $1983: 8$; $2006: 2-3$ ), ils seraient au nombre de 4000 aujourd'hui (Mélandri et Rovère, p. 46), peut-être en leur adjoignant leurs voisins et apparentés Nukuma. De remarquables ethnologues ont consacré à leurs coutumes et à leurs arts des études détaillées, qui en font, du point de vue occidental, une des sociétés les mieux connues du bassin du Sépik.

Fort différents dans leur volume et leur ambition, les deux ouvrages ici réunis ont pour sujet commun des 